\title{
Diabetic mastopathy: a case report
}

\author{
Carla Francisco*, Catarina Júlio, Ana Luísa Fontes, Inês Silveira Reis, Rosário Fernandes, \\ Sara Valadares, Pedro Sereno \\ Dr. Alfredo da Costa Maternity, Rua Viriato, 1069-089 Lisbon, Portugal
}

Received 7 August 2011; received in revised form 3 January 2012; accepted 3 January 2012

\begin{abstract}
Diabetic mastopathy (DMP) is an uncommon collection of clinical, radiological, and histological features, classically described in premenopausal women with long-term insulin-dependent diabetes mellitus. This entity can mimic breast carcinoma, but, in the appropriate clinical and imaging setting, the diagnosis can be made by core biopsy, avoiding unnecessary surgeries.

We report the case of a 34-year-old female, with a 12-year history of type 1 diabetes, who presented with bilateral breast lumps. Mammography, ultrasonography, and magnetic resonance imaging could not exclude the suspicion of malignancy, and a core biopsy was performed showing the typical histologic features of DMP.

The literature is briefly reviewed.

(C) 2012 Elsevier Inc. All rights reserved.
\end{abstract}

Keywords: Breast cancer; Diabetic mastopathy; Diabetes mellitus

\section{Introduction}

Diabetic mastopathy (DMP) is a collection of clinical, radiological, and histological features found in dense fibrous masses of the breast first described by Soler and Khardori [1] in 1984.

Since then, further studies have elucidated the histopathologic features of this unusual fibroinflammatory lesion that represents less than $1 \%$ of benign breast lesions [2].

The disease is associated with long-standing type 1 insulin-dependent diabetes mellitus (IDDM) but has also been reported in other autoimmune disorders and in type 2 diabetes mellitus $[3,4]$.

These patients present with solitary or multiple palpable, hard, painless, and irregular masses. Mammography usually discloses only dense glandular tissue, and ultrasound shows irregular, diffuse shadowing zones, sometimes hypoechoic attenuating nodules $[5,6]$. These clinical and imaging

\footnotetext{
* Corresponding author. 1070-234 Lisbon, Portugal.

E-mail address: carla.francisco@gmail.com (C. Francisco).
}

findings are inconclusive, and these lesions are often misdiagnosed as breast carcinomas.

The recognition of this rare but benign disease is crucial to avoid surgical biopsies which are not necessary [5].

\section{Case report}

The patient described is a 34-year-old female, with a 12year history of type 1 IDDM, who presented with bilateral breast lumps. There was no associated pain, but she noticed an increasing breast discomfort.

She was being treated for type 1 diabetes for the last 12 years and had retinopathy as secondary complication of diabetes.

Menarche had occurred at age 13, with regular menstrual cycles. For the last 12 months she used oral hormone contraceptive. She had two preterm vaginal deliveries and breastfeed for 6 months. Personal (besides IDDM) and familiar medical histories were unremarkable.

Physical examination revealed bilateral hard, irregular, mobile, painless nodules in the upper quadrants of the left 
breast and in the upper outer quadrant of the right breast. There was no nipple discharge, skin abnormalities, or axillary lymphadenopathy.

Mammography revealed bilateral heterogeneous dense breast parenchyma, BI RADS 4 of the ACR, with a nodular pattern, more pronounced in the upper quadrants. There were no suspicious microcalcifications, masses, or architectural distortions (Fig. 1).

The left breast ultrasound examination showed a hyperechoic mass with irregular contours, measuring $5 \times 2.5$ $\mathrm{cm}$ associated with intense posterior acoustic shadowing (Fig. 2).

A magnetic resonance imaging (MRI) was performed (1.5 $\mathrm{T}$ Sonata scanner, from Siemens) with the following pulse sequences: T1-weighted imaging in the axial plane, T2weighted and $\mathrm{T} 2$-weighted fast spin-echo imaging in the axial and sagittal plane, and dynamic 3D T1-weighted gradientecho MRI, before and after the intravenous administration of gadopentetate dimeglumine (Magnevist $0.1 \mathrm{mg} / \mathrm{kg}$ ), with five contrast-enhanced sequences being performed. Postprocessing analysis with subtraction images, multiplanar reconstruction in the axial plane, maximum-intensity-projection, and revision on a 3D workstation were also performed.

MRI detected symmetric heterogeneous low signal intensity images on T1 and T2. Subtraction images after contrast (gadolinium) intravenous administration showed poor and diffuse enhancement with no focal or nodular suspicious enhancement. Cinetic levels were nonspecific with late bilateral enhancement patterns (heterogeneous) due to parenchymal functional tissue (Fig. 3). The findings suggested a typically benign gradual type dynamic curve.
A

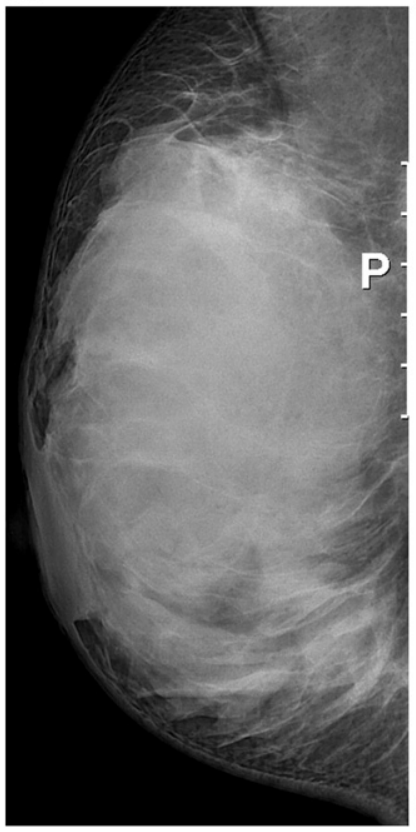

B

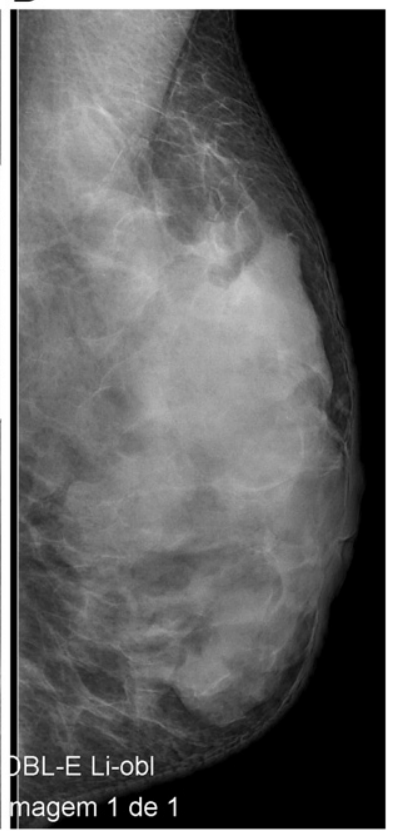

Fig. 1. (A, B) Bilateral mediolateral mammograms $\left(60^{\circ}\right)$. (A) Right breast. (B) Left breast. Dense heterogeneously fibroglandular pattern of breast parenchyma, with a nodular pattern (BI RADS 4 of the ACR).

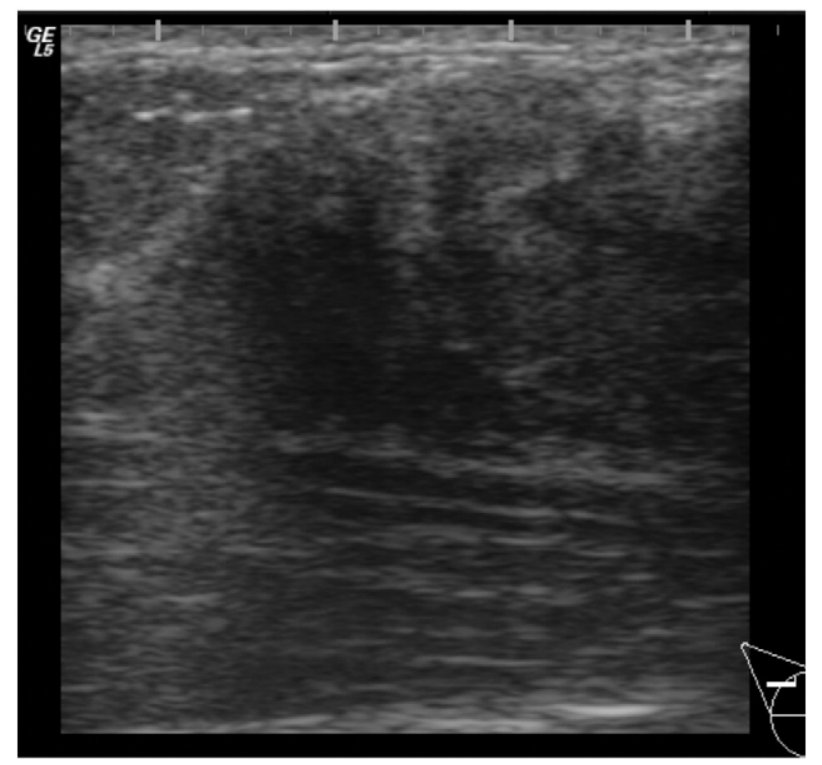

Fig. 2. Ultrasonographical image of the left breast reveals an irregular hyperechoic mass with intense posterior acoustic shadowing.

A biopsy under ultrasound guidance was performed. Histologic evaluation revealed a dense stromal fibrosis and some periductal lymphocytic infiltration without any evidence of atypia or malignancy suggestive of DMP (Fig. 4).

The patient remains in clinical and imaging surveillance, and currently has a follow-up of 2.5 years with a stable condition.

\section{Discussion}

Diabetic mastopathy (DMP) is a rare entity of selflimiting fibroinflammatory disease of the breast associated with type 1 diabetes mellitus. The prevalence of DMP has been found to be less than $1 \%$ of benign breast diseases, but can range from $0.6 \%$ to $13 \%$ in type 1 diabetic women [1,2].

Most published reports of DMP are of premenopausal women with long-standing type 1 IDDM, as in the case presented. Most patients with DMP also have complications arising from diabetes such as retinopathy, neuropathy, and nephropathy $[1,5]$.

This condition has also been described in type 2 diabetes and in patients with other endocrine disease, especially thyroid diseases. Rarely, it can also occur in men with longstanding diabetes $[5,7]$.

Clinical findings include hard, irregular, easily movable, painless breast masses. It can be solitary or multiple, unilateral or bilateral (in about $60 \%$ of cases), especially in the subareolar region. These characteristics raised the suspicion of carcinoma $[1,2,8]$. Our patient had a typical clinical presentation with bilateral masses.

Mammographic features usually show a localized increased density in the glandular pattern, with no distinct masses, spiculation, or calcifications. Strong acoustical 


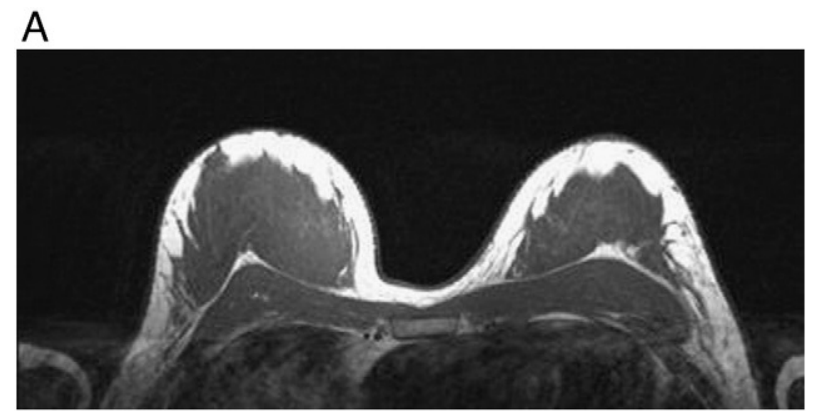

B

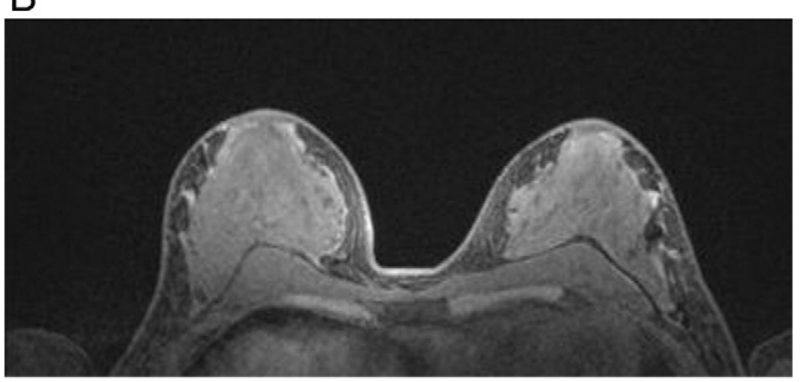

C

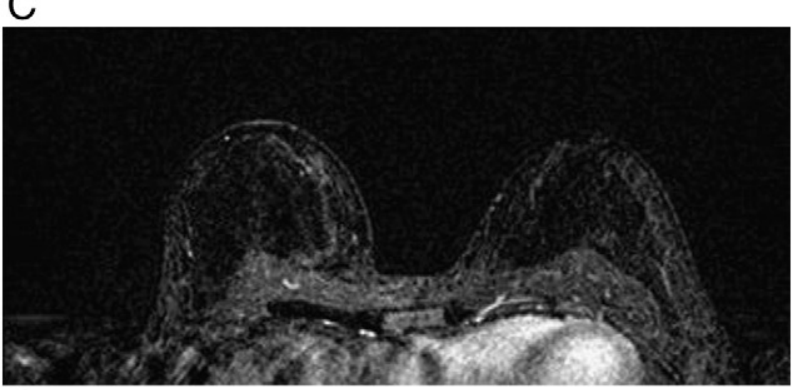

D

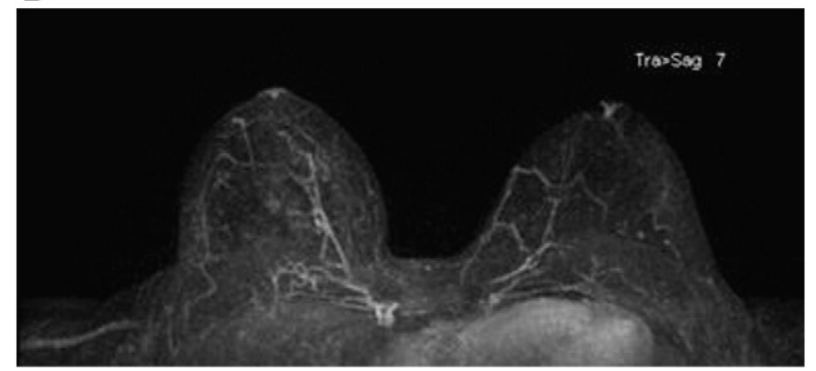

Fig. 3. (A-D) Axilal MR images. (A, B) Axial T1- and T2-weighted native sequences - low signal intensity images, symmetric features. (C) Axial T1weighted image, post-gadopentetate dimeglumine (Gd); subtraction 1' shows no focal or nodular suspicious enhancement. (D) Axial T1-weighted image, post Gd; subtraction $5^{\prime}$ shows late bilateral enhancement (heterogeneous) due to parenchymal functional tissue.

shadowing behind the palpable masses can be seen on sonography, consistent with our case report. Attenuation increases due to the fibrotic nature of the masses $[2,8]$.

Recent literature proposes that MRI features of DMP show the absence of enhancing mass with nonspecific parenchymal enhancement as opposed to carcinoma in which an avidly enhancing focal mass with irregular margins may be present. However, clinical and radiographic features are not specific to DMP and may be difficult to differentiate from malignancy without biopsy $[6,9,10]$.

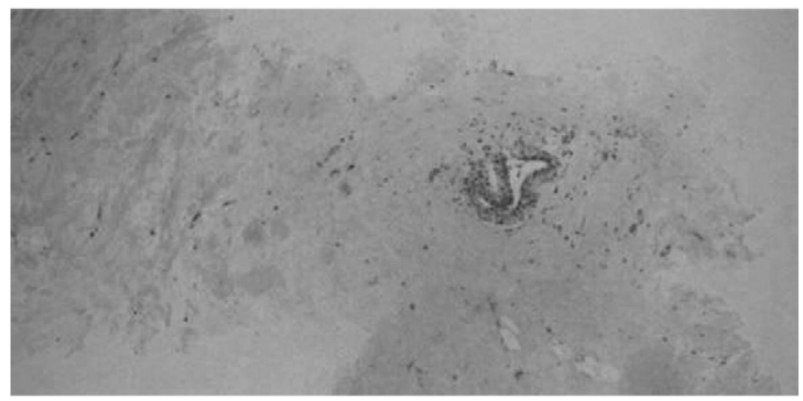

Fig. 4. Photomicrograph of core biopsy. Periductal lymphocytic infiltrate and stromal fibrosis (hematoxylin-eosin staining, original magnification $\times 20$ ).

Logan and Hoffman [11] have advocated the use of fine needle aspiration biopsy cytology in the diagnosis of DMP. They stated that the firm resistance experienced during the back-and-forward motion of the needle is stronger than that of other benign and malignant breast conditions; hence, this could be a clue to the diagnosis of DMP. Cytology usually yields benign ductal epithelium with a moderate amount of peculiar-appearing, dense, hyalinised fibrous tissue. Unfortunately, DMP contains little cellular material, making cytological diagnosis difficult.

Histologically, DMP lesions are composed of dense stromal keloid-like fibrosis containing little or no adipose tissue or cellular material. Furthermore, focal perivascular, periductal, and/or perilobular lymphocytic infiltrations with mature B-cell predominance are commonly seen. Epithelioid fibroblasts in the interlobular stroma are also frequently observed $[6,8,11]$. This correlates with our patient.

The awareness of the clinical presentation, the imaging, and cytological features are sufficient to diagnose DMP without the need for an excision biopsy. DMP tends to recur after surgical excision, in the same location, and involves more breast tissue than the preceding lesion, and therefore surgical biopsy should not be considered. In cases where the cytological diagnosis is difficult, core needle biopsy under ultrasound guidance avoids unnecessary surgical procedures that may exacerbate the condition $[2,5]$.

The pathogenesis of DMP is not completely understood, but several theories have been proposed considering a multifactorial etiology. Many researchers suggest a role for a secondary autoimmune reaction to abnormal extracellular matrix accumulation that is a manifestation of the effects of hyperglycemia on connective tissue. Hyperglycemia induces glycosylation, increased intermolecular cross-linkage, and matrix expansion that is altered in quantity and quality and resistant to degradation. The accumulation of abnormal matrix and advanced glycosylated end products acts as neoantigens and triggers an autoimmune response with B-cell proliferation and autoantibody production. Subsequent macrophage-mediated advanced glycosylation end-product removal and cytokine release may act as growth factors and induce additional matrix expansion and proliferation of collagen [2-4]. 
Since fibrous tissue deposition and collagen proliferation in the breast are similar to that of other diabetic complications, it is not unlikely that some patients with DMP will have thyroid, eye, and joint involvement due to their long-standing insulin-dependent diabetes [8].

Diabetic mastopathy has also been described in patients with type 2 diabetes mellitus who had been exposed to exogenous insulin. This suggests that the exogenous insulin may be related to its development. This may be due to inflammatory or immunologic reaction to the insulin, the vehicle, or a contaminant in the vehicle $[4,8]$.

The publications on DMP have been few and there is no standard protocol for the long-term management of these patients. If DMP is confirmed on histology, most reports recommend yearly physical examination, mammographic, and ultrasonographic examinations. However, as it has been noted that the number and size of DMP usually increase as the patient grows older, it is suggested that serial fine needle aspiration cytologies or core biopsies are performed on these new lesions to ensure that they are not malignant lesions. It is not recommended to perform open excisional biopsy if clinical and radiological features are suggestive of DMP [6].

There have been no reported cases of malignancy arising from DMP to the present date, and there has been one reported case of regression [8].

In conclusion, recognizing DMP requires awareness of the existence of this entity and a careful correlation of the patient's clinical history with the physical, radiological, and pathological examinations. DMP should be considered in the differential diagnosis of breast cancer but it is not a premalignant disease. As the disease can be managed conservatively but may recur, an accurate diagnosis is essential to avoid unwanted multiple surgical biopsies $[3,6]$.

\section{References}

[1] Soler NG, Khardori R. Fibrous disease of the breast, thyroiditis, and cheiroarthropathy in type I diabetes mellitus. Lancet 1984;1:193-5.

[2] Camuto P, Zetrenne E, Ponn T. Diabetic mastopathy: a report of 5 cases and a review of the literature. Arch Surg 2000;135:1190-3.

[3] Neetu G, Pathmanathan R, Weng NK. Diabetic mastopathy: a case report and literature review. Case Rep Oncol 2010;3:245-51.

[4] Tomaszewski JE, Brooks JS, Hicks D, Livolsi VA. Diabetic mastopathy: a distinctive clinicopathologic entity. Hum Pathol 1992; 23:780-6.

[5] Mak CW, et al. Case report-diabetic mastopathy. Br J Radiol 2003; 76:192-4.

[6] Pojchamarnwiputh S, Muttarak M, Na-ChiangMai W, Chaiwun B. Benign breast lesions mimicking carcinoma at mammography. Singapore Med J 2007;48(10):958-68.

[7] Akahori H, Kaneko M, Kiyohara K, Terahata S, Sugimoto T. A rare case of diabetic mastopathy in a Japanese man with type 2 diabetes Mellitus. Intern Med 2009;48(11):915-9.

[8] Keyoung JA, et al. Diabetic mastopathy. Applied Radiology 2003; 32(9):47-9.

[9] Wong KT, Tse GMK, Yang WT. Ultrasound and MR imaging of diabetic mastopathy. Clin Radiol 2002;57:730-5.

[10] Gabriel HA, Feng C, Mendelson EB, Benjamin S. Breast MRI for cancer detection in a patient with diabetic mastopathy. AJR Am J Roentgenol 2004;182(4):1081-3.

[11] Logan WW, Hoffman NY. Diabetic fibrous breast disease. Radiology 1989;172:667-70. 